\title{
Das organisationale Lerndreieck - eine lerntheoretische Perspektive auf organisationales Lernen
}

\author{
Henning Pätzold iD
}

Eingegangen: 12. Januar 2017 / Angenommen: 22. März 2017 / Online publiziert: 11. April 2017

(C) Der/die Autor(en) 2017. Dieser Artikel ist eine Open-Access-Publikation.

Zusammenfassung Wie wenig andere Bereiche der organisationswissenschaftlichen Literatur ist die Diskussion um organisationales Lernen von „Klassikern“ dominiert. Im vorliegenden Beitrag wird, nach einer knappen Diskussion der bisherigen Theorielage zum organisationalen Lernen, auf Grundlage des „Lerndreiecks“ von Knud Illeris - ein neues Modell organisationalen Lernens vorgestellt. Es nimmt die generelle Struktur dieses Dreiecks auf und transformiert aber die zentralen Konzepte von Illeris Modell in Richtung analoger Konzepte mit Bezug auf organisationale Prozesse, Strukturen und Eigenschaften. Weiterhin wird das Modell empirisch validiert, um zu zeigen, dass es zeitgemäße lerntheoretische Einsichten mit dem Konzept organisationalen Lernens in einer Weise verbindet, die über die „Klassiker“ hinausgehen. Die empirische Validierung geschieht mittels einer ethnografischen Feldstudie in einer NPO.

Schlüsselwörter Organisationales Lernen · Ethnografie · Organisationspädagogik · Lerntheorie

\section{The organizational learning triangle - Organizational learning from the perspective of learning theory}

\begin{abstract}
The reference to "classical" authors dominates the literature on organizational learning to an extent which is comparably large within the field of organizational sciences. After briefly reviewing the state of the art of theories of organizational learning, this article introduces a new model of organizational learning which builds on Knud Illeris' "learning triangle". Basically, it adopts the general
\end{abstract}

Unter Mitarbeit von Martin Müller, Universität Koblenz-Landau, Campus Koblenz

H. Pätzold ( $\square)$

Campus Koblenz, Universität Koblenz-Landau, Koblenz, Deutschland

E-Mail: paetzold@uni-koblenz.de 
structure of this triangle and transforms the crucial concepts of Illeris' approach to analogous concepts with respect to organizational processes, structures and characteristics. Further, the model is validated empirically in an ethnographic field study. Thus, it shall be demonstrated, that the model utilizes contemporary insights of learning theory with the concept of organizational learning in a way which goes beyond the "classical" approaches. The field study was conducted in a non-profit organization.

Keywords Organisational learning · Ethnography · Organizational education · Learning theory

Wie wenig andere Bereiche der organisationswissenschaftlichen Literatur ist die Diskussion um organisationales Lernen von „Klassikern“ dominiert, die innerhalb eines vergleichsweise kurzen Zeitraums einen Sachbereich beschrieben, strukturiert und über weite Strecken hinweg auch theoretisch dominiert haben. $\mathrm{Zu}$ nennen sind hier Autoren und Werke wie Cyert und March (1992), March und Olsen (1976), Argyris und Schön (1978) oder Senge (1990). Auch in jüngeren Lehrbüchern der betriebswirtschaftlich orientierten Organisationswissenschaft stehen im Kontext organisationalen Lernens Bezüge zu den genannten Klassikern im Mittelpunkt (vgl. Schreyögg und Geiger 2016, S. 357 ff.; Kieser und Walgenbach 2007, S. 437 f.; Berger und Bernhard-Mehlich 2006, S. 185). In der pädagogischen Auseinandersetzung gibt es zwar weiter gehende und eigenständige Entwürfe zum organisationalen Lernen (etwa bei Geißler 2000 oder Göhlich 2007), gleichwohl stellen sie aber auch in der erwachsenen- und organisationspädagogischen Diskussion bisher eher die Ausnahme dar (vgl. zum Überblick Pätzold 2015, S. 29 ff.). Vor diesem Hintergrund liegt es nahe, die erheblichen Fortschritte, die seither in der Lerntheorie erzielt worden sind, zu nutzen, um das Konzept organisationalen Lernens zu modernisieren. Ein Vorschlag hierzu liegt mit einer Bezugnahme auf die Lerntheorie von Knud Illeris vor, die sich hierfür eignet, insofern sie stark integrativ angelegt ist. Sie begreift sich weniger als Gegenentwurf zu bestehenden Theorien des Lernens, vielmehr versucht sie, in einem eigenständigen Modell viele Beiträge unterschiedlicher Lerntheorien miteinander $\mathrm{zu}$ vermitteln und so zu einem umfassenden Bild (menschlichen) Lernens zu gelangen (vgl. Illeris 2010). Illeris' Modell geht davon aus, dass individuelles Lernen nach außen hin in einer Interaktion mit der Umwelt besteht, der auf der „Innenseite“ ein kontinuierlicher Ausgleich zwischen einer kognitiven und einer emotionalen Dimension entspricht. Schon diese Verbindung eines (zweiseitigen) internen Prozess und eines Prozess der Interaktion mit der Umwelt in Illeris' Modell legt die Übertragung auf Organisationen nahe, insofern hier eine vergleichbare Differenzierung zwischen verschiedenen internen Prozessen und solchen, die die Außengrenze überschreiten, regelmäßig mit gedacht wird (vgl. z. B. Schreyögg und Geiger 2016, S. 19 ff.). Eine solche konzeptionelle Übertragung des Modells auf organisationales Lernen liegt vor (Pätzold 2017), eine empirische Anwendung steht bisher jedoch aus. Im vorliegenden Beitrag sollen deshalb auf Basis der Theorie gewonnene Befunde einer ethnografischen Feldstudie zum organisationalen Lernen in einer kleinen und jungen Non-Profit-Organisation innerhalb 
des Handlungsfelds sozial-ökologischer Gründeraktivitäten dargestellt werden. Die hierauf bezogene, erste Forschungsfrage lautet: Erfüllt das auf Grundlage der Lerntheorie Illeris' formulierte Modell organisationalen Lernens die Anforderungen, die an eine theoretische Modellierung grundsätzlich zu stellen sind?

Ein weiteres Merkmal der Diskussion um organisationales Lernen liegt in der kontrovers diskutierten Frage, inwiefern es sinnvoll ist, Organisationen als lernende Akteure eigener Art und damit den organisationalen Lernvorgang als eigenständiges Phänomen zu verstehen, das nicht gänzlich in der Kollektivierung individueller Lernprozesse der Organisationsmitglieder aufgeht (vgl. hierzu im Überblick Behrmann 2010; kritisch Faulstich 2013, S. 192 ff.). Eine Theorie organisationalen Lernens sollte - in der Tradition lerntheoretischer Entwürfe - in der Lage sein, einen Akteur zu benennen, dem der Lernprozess zugeschrieben wird. Die Reduktion des organisationalen Lernens auf die Aktivitäten der Mitglieder liegt einerseits nahe, insofern Lernen in der Regel zunächst vom Menschen (oder allgemeiner: Organismus) aus gedacht wird, andererseits weist sie strukturell ähnliche Probleme auf, wie die Reduktion menschlicher Lernvorgänge auf Prozesse des Gehirns (vgl. Meyer-Drawe 2008, S. 41). Hieraus ergibt sich die zweite Forschungsfrage: Liefert das Modell eine angemessene Abstraktion des Lernvorgangs von den beteiligten Individuen, die eine Konzeption der Organisation als eigenständig lernendem Akteur ermöglicht und rechtfertigt?

\section{Organisationales Lernen: Zum Stand der Modellbildung}

\subsection{Eckpfeiler und Entwicklungen der Theorie organisationalen Lernens}

Organisationales Lernen wird in der organisationswissenschaftlichen Literatur schwerpunktmäßig entlang traditionsreicher Konzepte des Lernens modelliert. Zwar lassen sich auch hier Vorläufer und Ideengeber aus der ferneren Vergangenheit identifizieren, dennoch sind die Bezüge vergleichsweise lose. Die Klassiker ihrerseits lassen sich durch ihre eigenen lerntheoretischen Bezüge recht klar beschreiben: Am Anfang der Debatte stehen Beiträge von March, Olsen und anderen (March und Olsen 1976). Sie sind im Umfeld einer stark durch die Erfolge der behavioristischen Psychologie geprägten Konzeption organisationaler Vorgänge angesiedelt, in der Variationen des Reiz-Reaktions-Schemas mit Annahmen über die Kognitionen von Organisationsmitgliedern verbunden werden (vgl. March und Simon 1958). Die lerntheoretischen Grundlagen, mit denen hier gearbeitet wird, sind überwiegend in der ersten Hälfte bzw. um die Mitte des 20. Jahrhunderts dargestellt worden. Ein weiteres herausragendes Werk ist „Organizational Learning“ von Argyris und Schön (1978). Eine wesentliche, immer wieder aufgegriffene Idee des Werkes ist das Mehrschleifenmodell des Lernens, das die Autoren auf die Begriffe ,single-looplearning“, „double-loop-learning“ und ,deutero-learning“ bringen (ebd., S. 2 f.), wobei darauf hingewiesen wird, dass das Modell unmittelbar Gregory Batesons „logischen Kategorien von Lernen und Kommunikation“ (Bateson 1985, S. 362 ff., zum Deuterolernen auch ebd., S. 378 und S. 229) nachempfunden ist, die ursprünglich ebenfalls um die Mitte des 20. Jahrhunderts dargestellt wurden. Mit 
Peter Senges „fifth discipline“ (Senge 1990) ist schließlich eine dritte Referenz organisationalen Lernens zu nennen, die sich von den anderen jedoch insofern unterscheidet, als sie deutlich praxeologischer angelegt und in ihren Theoriebezügen weniger klar ist. Senge verfolgt eine Verbindung kognitiver und systemtheoretischer Perspektiven, nimmt dabei aber durchaus schwer wiegende theoretische Aporien und Widersprüche in Kauf (vgl. Leichthammer 2000, S. 285). Wie in der Einleitung bereits dargestellt, beziehen sich zahlreiche aktuelle Einführungstexte wie auch Forschungsarbeiten aus der Organisationswissenschaft und der Pädagogik auf diese Werke und machen sie zur Grundlage von Forschungen und Konzeptionen organisationalen Lernens. Zentral für Konzepte organisationalen Lernens sind demzufolge auf lerntheoretischer Ebene insbesondere der Behaviorismus, Batesons logische Kategorien sowie der Kognitivismus piagetscher Prägung und teilweise Weiterentwicklungen (etwa durch Kolb 1984). Insbesondere die Bezüge zum Kognitivismus bieten auch Anschlussmöglichkeiten für konstruktivistische und systemtheoretische Konzepte, ohne dass diese allerdings - von wenigen Ausnahmen abgesehen - in lerntheoretischer Hinsicht zu substanziellen Weiterentwicklungen führen würden. ${ }^{1}$

Natürlich ist die organisationswissenschaftliche Forschung und Theoriebildung hier nicht stehen geblieben. Die weitere Entwicklung hat sich jedoch weniger den theoretischen Grundlagen gewidmet, als Fragen der Gestaltbarkeit und Technisierbarkeit. Indem organisationales Lernen als nützlicher, wenn nicht sogar notwendiger Prozess vorausgesetzt wurde, gerieten Herausforderungen der konkreten Ausgestaltung in den Blick. Die behavioristisch-kognitivistische Prägung des Lernbegriffs äußerte sich folgerichtigerweise in der Konzentration auf Prozesse des Wissensmanagements (vgl. Fahrenwald 2009) und die Gestaltung lernförderlicher Arbeitsumgebungen, etwa in Communities of Practice (vgl. zum Überblick Mandl et al. 2004; auch Schrader und Berzbach 2005, S. 2 f.).

\subsection{Pädagogische Transformationen und Rekonfigurationen}

Auch in der pädagogischen Debatte ist das organisationale Lernen aufgegriffen worden - einerseits insofern Lernen ein ,pädagogischer Grundbegriff“ (Göhlich und Zirfas 2007) ist, andererseits mit dem Entstehen der Organisationspädagogik als eigenständiger Teildisziplin (Göhlich et al. 2014). Mit dem Werk „Organisationspädagogik" (Geißler 2000) liegt ein umfassender und bis heute maßgeblicher Entwurf einer pädagogischen Konzeption organisationalen Geschehens vor. Bezüglich des Lernbegriffs kann hier - gegenüber den oben genannten Zugängen - von einer Transformation gesprochen werden, insofern Geißler diese und andere „Klassiker“ zwar aufgreift, deren Blick auf Lernen aber durch die pädagogische Perspektive der Bildung neu ordnet (ebd., S. 7). Vor diesem Hintergrund entsteht ein Strukturmodell organisationalen Lernens, das „operatives Anpassungslernen“, ,strategisches Erschließungslernen“ und „normatives Identitätslernen“ (ebd., S. 51) unterscheidet. Auch Geißler schließt hier an Batesons ,deutero-learning“ an (ebd., S. 50), geht

\footnotetext{
${ }^{1}$ Ein Grund dafür mag darin liegen, dass systemtheoretische und konstruktivistische Konzepte eine hohe Affinität zu evolutionären Logiken (vgl. Schurz 2011) aufweisen und es damit nahe legen, organisationale Veränderungsprozesse generell als Entwicklung zu konzipieren, so etwa bei Schiersmann und Thiel (2014).
} 
jedoch durch die normative und identitätsbezogene Perspektive deutlich darüber hinaus. Sein Beitrag stellt einen wichtigen Schritt in der Konzeption organisationaler Lernvorgänge aus pädagogischer Perspektive dar. Mit der Nutzung der Kategorie Bildung stärkt er den pädagogischen Bezug, nimmt aber zugleich eine geringere internationale Anschlussfähigkeit in Kauf, insofern der Bildungsdiskurs außerhalb der deutschsprachigen Pädagogik nur wenig Resonanz findet. Hierauf weisen auch Göhlich und Zirfas (2007, S. 87) hin, stellen aber zugleich klar, dass der Aufbau des Bildungsbegriffs um den Preis einer Verflachung des Lernbegriffs erkauft wurde (vgl. ebd., S. 87 ff.), der so in anderen Sprachen nicht stattgefunden hat. Göhlich liefert einen weiteren pädagogischen Vorschlag zur Konzeption von organisationalem Lernen als „Erwerb, Aufbau, Einbau und Umbau von Wissen durch Organisationen" (Göhlich 2007, S. 224), der die kognitive Verengung der vorliegenden Beiträge überwinden soll. Für ihn ist Lernen primär ein ,,mustermimetischer Prozess" (ebd., S. 225), ohne dass diese Mimesis jeweils in kognitiven Prozessen aufgehen oder durch diese vollständig determiniert sein müsse. Sein Ansatz stellt in gewissem Sinne eine Rekonfiguration bisheriger Konzepte dar, insofern er deren kognitive Schwerpunktsetzung revidiert und die Dimensionen Wissen, Können, Leben und Lernen als gleichwertig in sein Konzept aufnimmt.

Den Ansätzen ist gemein, dass sie über die bisherigen Modelle zum organisationalen Lernen hinausgehen und pädagogische Perspektiven erschließen. Gleichzeitig werfen sie damit sehr grundlegende neue Fragen auf, die sie im Kontext empirischer Arbeit nicht unbedingt einfach handhabbar machen (obwohl durchaus an sie angeschlossen wird, vgl. Pätzold 2015, S. 29 f.). Als Alternative zur pädagogischen informierten Transformation und Rekonfiguration der Theorien organisationalen Lernens erscheint deshalb deren Modernisierung. Entwicklungen im Bereich der (pädagogischen) Lerntheorien können genutzt werden, um organisationales Lernen, analog zu seinen Ausgangsbegründungen, unter Rückgriff auf entsprechende Theorieangebote zu fassen. Im Folgenden werden zentrale Ideen dieses Versuchs im Anschluss an eine eigene Arbeit hierzu (Pätzold 2017) skizziert, um den Entwurf dann hinsichtlich seiner empirischen Plausibilität und Anwendbarkeit zu erproben.

\subsection{Das Lerndreieck organisationalen Lernens}

Im Mittelpunkt der Arbeiten von Knud Illeris steht ein Konzept des Lernens, das drei Dimensionen unterscheidet, die - bei teilweise unterschiedlichen Benennungen im Detail - immer einerseits ein Wechselspiel zwischen kognitiven und emotionalen Aspekten des Lernens und andererseits eine Wechselwirkung zwischen individuellem Lernen und Umwelt adressieren (vgl. Illeris 2003, 2004, 2007, 2010). Das Modell gewinnt er einerseits aus der Reflexion beobachtbarer oder beschreibbarer Lernvorgänge, andererseits aus der eklektizistischen Auseinandersetzung mit wesentlichen Beiträgen aus der gesamten Geschichte (westlicher) Lerntheorie und Lernforschung (vgl. auch Baumgartner 2008, S. 474). Gerade das eklektizistische Vorgehen ist nicht unproblematisch, insofern die jeweiligen Bezugstheorien oft weit auseinanderliegen und teilweise durchaus als Gegenentwürfe zueinander gelesen werden können. Die Leistung Illeris' besteht aber gerade darin, diese Widersprüchlichkeit gewissermaßen als Spannung in sein Modell einzubauen, die nicht aufgelöst 
Abb. 1 Lerndreieck organisationalen Lernens

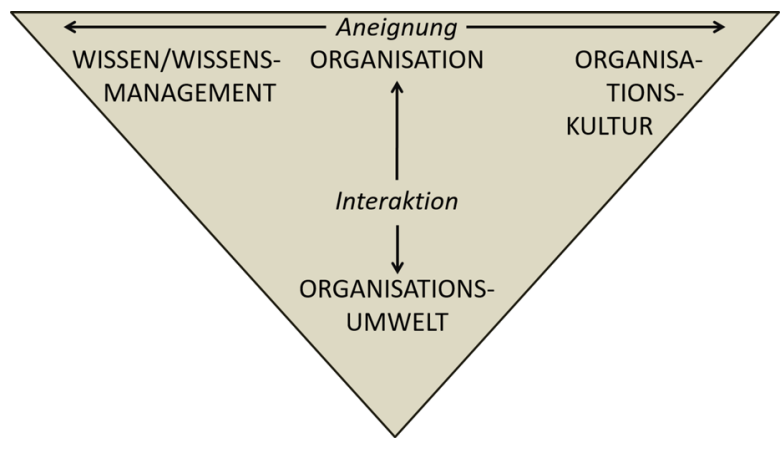

werden kann, aber dynamisch bearbeitet wird. So wird mit der ,horizontalen“ Dimension von Kognition und Emotion gerade herausgearbeitet, dass eine Erklärung menschlichen Lernens weder ein Primat der Kognition noch eines der Emotion postulieren kann, sondern stets den dynamischen Ausgleich zwischen beiden bedenken muss. Jenseits einer Würdigung der Theorie (vgl. hierzu auch Mott 2009; Baumgartner 2008) soll an dieser Stelle knapp deren Übertragung auf organisationales Lernen skizziert werden, wie sie der empirischen Untersuchung zugrunde gelegt wurde.

Illeris unterscheidet, wie angesprochen, die Dimensionen Emotion, Kognition und Umwelt. Zwischen ersteren oszilliert ein Prozess, den er der Person als Akteur zuschreibt und als Aneignung bezeichnet. Die Person befindet sich wiederum in einem Prozess der Interaktion mit der Umwelt. Das Modell wurde in verschiedenen Publikationen in Form eines Dreiecks dargestellt (z. B. Illeris 2004, S. 95; in deutscher Übersetzung u. a. in Illeris 2006, S. 31). Für die vorliegende Untersuchung wurde $n$ die Stelle der Person als lernendem Akteur die Organisation gesetzt und eine Übertragung der jeweiligen Begriffe aus Illeris' Dreieck auf die Bedingungen und Vorgänge in Organisationen vorgenommen. Ziel war es, dabei an bestehende organisationswissenschaftliche Konzepte anzuschließen, denn die hier thematisieren innerorganisationalen Wechselverhältnisse sowie diejenigen mit der Umwelt weisen erkennbar Parallelen auf. So findet sich, beginnend spätestens mit der HumanRelations-Debatte immer wieder der Hinweis, dass organisationale Vorgänge nicht als unverfälschte Umsetzung einer Rationalität (oder auch nur einer Regelhaftigkeit) betrachtet werden können (genauer vgl. Pätzold 2017). Weiter reichend kontextualisiert wurden derartige Beobachtungen dann mit dem Begriff der Organisationskultur (vgl. Schein 2004). Das Resultat der vorgenommenen Übertragung ist das in Abb. 1 dargestellte Lerndreieck organisationalen Lernens.

Mit der Übertragung liegt ein Versuch vor, einen Beitrag zur Theorie organisationalen Lernens auf der Grundlage einer zeitgenössischen Lerntheorie zu konzipieren. Ein nächster Schritt besteht nun darin, diesen Beitrag auf seine empirische Anschlussfähigkeit im Sinne der oben formulierten Forschungsfragen zu überprüfen. In welchem Umfang erfüllt das Modell einerseits die Anforderungen, die an eine Theorie organisationalen Lernens zu stellen sind und liefert es andererseits eine konsistente Abstraktion organisationalen Lernens von individuellen Akteuren, die es schlüssig erscheinen lässt, von der Organisation als lernendem Akteur zu sprechen? 


\section{Empirische Untersuchung}

\subsection{Methodisches Vorgehen}

Mit dem Lerndreieck organisationalen Lernens liegt ein Entwurf vor, wie ein bestimmter Bereich organisationaler Vorgänge als organisationales Lernen abgegrenzt und intern weiter differenziert werden kann. Im Sinne einer Theorie sollte er einen Beitrag dazu leisten, einen Gegenstandsbereich durch systematisch aufeinander bezogene Begriffe und widerspruchsfreie Aussagen über deren Beziehungen für die sachliche Betrachtung und empirische Analyse zu erschließen (vgl. Kromrey 1998, S. 48). Im vorliegenden Fall bewegen wir uns in einem eher explorativ zu erschlieBenden Feld, insofern der Bestand an umfassenden, empirisch anschlussfähigen Theorien zum organisationalen Lernen nicht groß ist. Es geht also (noch) nicht darum, einzelne Hypothesen abzuleiten und zu überprüfen, sondern zunächst um den Erweis, dass die begriffliche Ordnung, die das organisationale Lerndreieck umreißt, ein sinnvoller Ausgangspunkt zur Anwendung ,wissenschaftlicher Verfahren der Wirklichkeitsanalyse“ (Gruschka 2006, S. 36) ist. Dem explorativen Charakter wurde methodisch durch ein ethnografisches Vorgehen entsprochen (vgl. Thomas 2010). Im Mittelpunkt stand, nach einer vorausgehenden Analyse der Organisation entlang vorliegender Dokumente, eine 14-tägige systematische Felderkundung im Wege teilnehmender Beobachtung. Der Mitautor hat hierzu ein Praktikum in der Einrichtung absolviert, das sowohl die Möglichkeit bot, umfassend Merkmale und Artefakte der Einrichtung zu dokumentieren, als auch Gespräche mit Mitgliedern der Organisation zu führen, an typischen Arbeitsprozessen teilzunehmen und die Aktivitäten anderer Organisationsmitglieder zu beobachten. Die Beobachtung ist dabei insofern als offen zu bezeichnen, als die Forschungsintention im Rahmen des Praktikums bekannt gemacht wurde - gleichzeitig ist sie in der alltäglichen Interaktion aber im Regelfall den Interaktionspartnern kaum erkennbar bewusst gewesen. Im Rahmen der Feldphase wurden umfangreiche und unterschiedliche Daten gesammelt, die teilweise für die Auswertung weiter aufbereitet und aggregiert wurden. Neben 139 Fotografien, drei Interviewaufzeichnungen sowie den Feldnotizen liegt u. a. die interviewgestützte Darstellung einer egozentrischen Netzwerkkarte der Organisation vor. ${ }^{2}$

Zur Auswertung der Daten wurden die analytischen Dimensionen „Wissen“, „Organisationskultur“ und „Umwelt“ (vgl. Abb. 1) unter Berücksichtigung ihrer ursprünglichen Herkunft bei Illeris genutzt, um zentrale Beobachtungen der Feldphase zu klassifizieren. Im Wege der qualitativen Inhaltsanalyse (Mayring 2010) wurden entsprechende textlich vorliegende Dokumente (Interviews, Feldnotizen) verdichtet. Ergänzend wurden Bilddokumente genutzt, um Notizen aus den Feldbeobachtungen zu prüfen. Die Klassifizierung erwies sich als recht trennscharf, wobei ca. ein Drittel der Beobachtungen mehreren Dimensionen zugeordnet wurde. Die Beobachtungen zur Dimension „Umwelt“ wurden im Zusammenhang mit der Erstellung der Netzwerkkarte verwendet und deshalb hier noch nicht berücksichtigt. Tab. 1 stellt

\footnotetext{
2 Auf die Darstellung der Netzwerkkarte muss zugunsten der Wahrung der Interessen der hierin dargestellten Personen und Organisationen verzichtet werden.
} 
Tab. 1 Zuordnung von Beobachtungen zu den Kategorien „Umgang mit Wissen“ und „Organisationskultur"

\begin{tabular}{ll}
\hline Umgang mit Wissen (Inhalt) & Organisationskultur (Antrieb) \\
\hline $\begin{array}{l}\text { Aufgaben/Aufträge werden detailliert beschrieben } \\
\text { und erklärt }\end{array}$ & $\begin{array}{l}\text { Wir sind eine Gemeinschaft vs. jeder für sich (Ge- } \\
\text { meinschaftsküche, in der kaum gemeinschaftliche } \\
\text { Aktivität stattfindet) } \\
\text { Ökologisches Bewusstsein (z. B. Papierverbrauch } \\
\text { Visuelle Darstellung von Wissen hat große Bedeu- } \\
\text { tung (visual thinking) }\end{array}$ \\
$\begin{array}{l}\text { Wissen/Informationen werden auch gezielt zurück } \\
\text { gehalten (z. B. Abhängen bestimmter Plakate bei }\end{array}$ & $\begin{array}{l}\text { Flexibilität und Lockerheit (z. B. keine festen } \\
\text { Arbeitsplätze und Arbeitszeiten) }\end{array}$ \\
$\begin{array}{l}\text { Rigitale Ordnerstruktur komplex, nicht für jeden } \\
\text { Mitarbeiter verständlich oder zugänglich }\end{array}$ & ... \\
\hline ... & \\
\hline
\end{tabular}

beispielhaft einige Beobachtungen und ihre Zuordnung zu den jeweiligen Dimensionen dar.

\subsection{Auswertung und Ergebnisse}

Die oben dargestellte Aufbereitung der Daten handelt von allgemeinen Feldbeobachtungen. Anders als etwa bei Boreham und Morgan (2004) wurden nicht Praktiken organisationalen Lernens beobachtet, sondern organisationale Merkmale und Vorgänge, die erst im Rahmen der Auswertung mit organisationalem Lernen in Verbindung gebracht wurden. Dabei spielte die auch bei Illeris bedeutsame Vorstellung von Lernen als Umgang mit Umwelt eine zentrale Rolle. Damit verbunden ist die Erkenntnis, dass Lernen als Ereignis kaum beobachtet werden kann, wohl aber Vorgänge, die auf Lernen schließen lassen - ein Sachverhalt, der eine Theorie organisationalen Lernens mit Theorien des individuellen Lernens verbindet. Nachdem der erste Schritt der Datenaufbereitung bereits gezeigt hat, dass die Dimensionen des Lerndreiecks eine Strukturierung der Feldbeobachtungen liefern, die organisationale Beobachtungen als relevant für jeweils bestimmte Dimensionen eines Lernprozesses auszuweisen vermögen, bestand der nächste Schritt darin, auf dieser Grundlage hypothetische Aussagen zu Lernprozessen zu formulieren. Hierzu wurden die dynamischen Aspekte des Modells genutzt, die Vermittlung zwischen Inhalt und Antrieb (bzw. Umgang mit Wissen und Organisationskultur) im Prozess der Aneignung und die Vermittlung zwischen Organisation und Organisationsumwelt im Prozess der Interaktion. Als Maßstab sollte gelten, ob sich hypothetische Zusammenhangsaussagen formulieren lassen, die empirisch überprüfbar sind und Ansatzpunkte für Interventionen, etwa im Sinne einer Organisationsberatung, bieten. ${ }^{3}$

\footnotetext{
3 Faulstich und Grell haben gegenüber pädagogischen Lerntheorien unter anderem den aus Sicht der Autoren berechtigten Anspruch formuliert, dass sich in ihrem Kontext ein adäquates Konzept von Lehre entwickeln lassen muss (vgl. Faulstich et al. 2005, S. 21). Die Nutzbarkeit einer Theorie organisationalen Lernens im Sinne etwa der Organisationsberatung/-entwicklung nimmt diesen Anspruch auf.
} 


\subsection{Vermittlung}

Als markant erwies sich der Blick auf den Ausgleich von Kognition und Antrieb (bzw. Umgang mit Wissen und Organisationskultur) im Rahmen der Aneignung. An zentralen Stellen zeigte sich bei der untersuchten Organisation ein Übergewicht zugunsten der Antriebsseite: Hier werden organisationskulturelle Standards gesetzt und praktiziert, andererseits werden sie auf der Seite des Umgangs mit Wissen nicht eingeholt, wodurch Schwierigkeiten entstehen. So wird eine große Flexibilität angestrebt und es wird aus ökologischen Gründen weitgehend auf Papierdokumentation verzichtet. Da gleichzeitig eine unklare Ablagestruktur auf den Computerlaufwerken herrscht, können notwendige Informationen jedoch oft nicht gefunden werden. In Einzelfällen kann nicht rekonstruiert werden, welches der Organisationsmitglieder eine Unterschrift geleistet hat. Über das grundlegende Organisationsziel herrscht weitgehende Einigkeit und es hat auch eine verhaltenssteuernde Ausstrahlung auf die Mitglieder, es bleiben aber auch wesentliche Aspekte der Gewichtung von Teilzielen unklar. So ist auf der Ebene des Antriebs klar, dass diese Ziele erreicht werden sollen, gleichzeitig bleibt auf der Ebene des Umgangs mit Wissen unklar, wie im Falle von Zielkonflikten zwischen den Zielen sachlich priorisiert werden soll. Diese Beobachtungen sind nicht als Mängeldiagnose der Organisation zu verstehen, im Gegenteil: Auf der Seite der Organisationskultur zeigt sich ein starker und vielschichtiger Antrieb, der es den Organisationsmitgliedern erlaubt, persönliche Ziele und Ziele der Organisation fruchtbar miteinander zu verbinden, insofern sie sich bewusst für die Arbeit in einer ökologisch-sozial orientierten Organisation entschieden haben. Diese Beobachtung steht im Einklang mit der Erwartung, dass Non-ProfitEinrichtungen hinsichtlich der Harmonisierung von individuellen und organisationalen Werten eine oft gute Ausgangsposition haben (vgl. Pätzold 2017; Schröer 2011, S. 103 ff.). Für die weitere Organisationsentwicklung ergibt sich jedoch die Aufgabe, diesen „Vorsprung“ auf der Seite des Antriebs durch eine entsprechende Praxis auf der Seite des Umgangs mit Wissen einzuholen. Die Untersuchung liefert hier konkrete Hinweise, etwa in Bezug auf Verfahren und Medien der Dokumentation, aber auch auf das Potenzial, das in einer grundlegenderen Klärung von Abläufen, etwa bei Zielkonflikten, liegt.

\subsection{Interaktion}

In der Netzwerkdarstellung, die Bezüge zwischen der Organisation selbst und benachbarten Akteuren (insbesondere Organisationen, aber auch Personen) herausstellt, wird eine große Komplexität erkennbar. Viele andere Akteure sind für die untersuchte Organisation relevant und sie sind es aus sehr unterschiedlichen Gründen. Teilweise nehmen sie Leistungen der Organisation in Anspruch, teilweise stellen sie notwendige Ressourcen bereit, in vielen Fällen sind sie aber auch deshalb relevant, weil die Organisation in Zukunft den Kontakt formalisieren und im Wege der Kooperation nutzen möchte (das gilt etwa für viele der wissenschaftlichen Organisationen im Netzwerk). Schließlich gibt es eine zentrale Person, die neben der untersuchten Organisation weitere gegründet hat. Zwischen diesen gibt es Kontakte, aber natürlich auch potenzielle Konkurrenzverhältnisse (insbesondere bezüglich 
der Zuordnung von Aufgaben und Ressourcen). Eine Herausforderung liegt auch hier darin, dass die Gründungsideen, die auf der Seite des Antriebs verortet werden können, in einem Gefüge von Organisationen verteilt werden müssen, was sich Organisationskulturell nicht leicht abbilden lässt: Eine ideelle Orientierung (wie etwa ökologisches Handeln) lässt sich nicht in gleicher Weise schlüssig in verschiedene Teiloperationen zerlegen (bzw. auf Organisationen verteilen), wie etwa ein Montageprozess. Auch die Interaktion mit der Umwelt wird so aus der Perspektive von Organisationskultur und Umgang mit Wissen unterschiedlich akzentuiert. So spielen manche Organisationen aus der Umwelt als Informationsquellen eine wichtige Rolle, andere eher, weil der Kontakt aus Sicht der ideellen Orientierung für wichtig erachtet wird.

Darüber hinaus zeigt das organisationale Feld eine für eine kleine Organisation nicht leicht beherrschbare Vielfalt aus Einzelpersonen und Organisationen, die für sehr unterschiedliche Leistungen und Orientierungen stehen. Aus der Perspektive des Umgangs mit Wissen erscheint es sinnvoll, die Organisationskontakte stärker zu strukturieren, eventuell auch zu formalisieren oder sogar auszudünnen. Gleichzeitig erscheint der ideelle Wert von Kontakten zu bestimmten Organisationen als ein Potenzial, das möglicherweise noch stärker genutzt werden könnte, insofern eine Kooperation unter der Voraussetzung ähnlicher und einander förderlicher Ziele weniger Aufwand benötigt als Kooperationen unter der Bedingung divergierender Ziele oder Konkurrenz.

\section{Fazit}

Die ethnografische Feldstudie brachte im Rahmen der vorliegenden Untersuchung Beobachtungen hervor, die sich entlang des Modells organisationalen Lernens (vgl. Abb. 1) strukturieren lassen. Die Kategorien Umgang mit Wissen, Organisationskultur und Organisationsumwelt sowie Vermittlung und Interaktion können genutzt werden, um Phänomene in Organisationen in Bezug auf organisationales Lernen zu ordnen. Sie erweisen sich dabei als recht überschneidungsarm und gleichzeitig ausreichend, um die im ethnographischen Zugang aufgezeichneten Beobachtungen vollständig zu erfassen. So erlauben sie eine strukturierte Betrachtung der Organisation, die Ausgangspunkt sowohl für weitere theoretische Überlegungen als auch für praktische Optionen der Organisationsentwicklung darstellt. Der Vorgang des Lernens konnte dabei konsequent auf der Ebene der Organisation verortet werden. Die individuellen Vorgänge, die beobachtet und aufgezeichnet wurden, waren sehr unterschiedlicher Art, und während sie auf organisationaler Ebene Aspekten des Lernens zugeordnet werden konnten, waren sie auf individueller Ebene mitunter gänzlich anderer Natur (beispielsweise: Papier vermeiden). Die Betrachtungsweise ermöglichte insofern auch die eingeforderte Abstraktion organisationalen Lernens von Lernvorgängen auf individueller Ebene.

Die explorative Anlage der Studie erlaubt allerdings keine endgültige Aussage über die Passung und Eignung des Modells, sie legt diese lediglich nahe. Überdies nutzt sie bisher nur einen Teil des Potenzials, das durch die Übertragung zeitgemäßer Theorien des Lernens auf Organisationen besteht. Schon innerhalb der Theorie 
Illeris' gäbe es weitere Anschlussmöglichkeiten, insbesondere die (auch in anderen Lerntheorien) thematisierte Stufung von Lernprozessen unterschiedlicher Tiefe (vgl. Pätzold 2011, S. 18 ff.). ${ }^{4}$ Im Gegensatz zu bereits vorliegenden Vorschlägen hierzu (insbesondere dem des Ein- bzw. Mehrschleifenlernens bei Argyris und Schön) werden die Lernebenen hier weniger nach ihrer logischen Abfolge thematisiert, als entlang des Aufwandes, der erforderlich ist, damit ein Lernprozess in Gang kommen kann. Weiterhin ist zu problematisieren, dass Illeris' Modell ein wenig differenziertes Verhältnis zur Leiblichkeit von Lernprozessen hat. Innerhalb der Lerntheorien gibt es hier deutlich avanciertere Vorschläge etwa aus der Perspektive der Phänomenologie. Unter der Überschrift ,,der Einspruch der Dinge“ (Meyer-Drawe 2008, S. 159) eröffnet Meyer-Drawe hier bereits eine Perspektive, die auch für organisationales Lernen ertragreich erscheint, insofern allgemeiner die Materialität als relevante Kategorie eigener Art aufgegriffen werden kann. Soziomaterielle Untersuchungen in der Erziehungswissenschaft (vgl. Fenwick et al. 2011) und speziell in der Organisationspädagogik (vgl. Pätzold 2016) liefern hierzu Vorarbeiten und Anknüpfungspunkte.

Open Access Dieser Artikel wird unter der Creative Commons Namensnennung 4.0 International Lizenz (http://creativecommons.org/licenses/by/4.0/deed.de) veröffentlicht, welche die Nutzung, Vervielfältigung, Bearbeitung, Verbreitung und Wiedergabe in jeglichem Medium und Format erlaubt, sofern Sie den/die ursprünglichen Autor(en) und die Quelle ordnungsgemäß nennen, einen Link zur Creative Commons Lizenz beifügen und angeben, ob Änderungen vorgenommen wurden.

\section{Literatur}

Argyris, C., \& Schön, D. A. (1978). Organizational learning. Reading, Mass: Addison-Wesley Pub. Co. Bateson, G. (1985). Ökologie des Geistes. Frankfurt a.M.: Suhrkamp.

Baumgartner, L. M. (2008). How we learn: Learning and non-learning in school and beyond. Human Resource Development Review, 7(4), 472-475. Book Review: Illeris, K. (2007).

Behrmann, D. (2010). Lernen in der Organisation - Rekonstruktionen zum Verhältnis von individuellem und organisationalem Lernen. In C. Heidsiek \& J. Petersen (Hrsg.), Organisationslernen im 21. Jahrhundert. Festschrift für Harald Geißler (S. 93-103). Frankfurt, a.M. u. a.: Peter Lang.

Berger, U., \& Bernhard-Mehlich, I. (2006). Die Verhaltenswissenschaftliche Entscheidungstheorie. In A. Kieser \& M. Ebers (Hrsg.), Organisationstheorien (6. Aufl. S. 169-214). Stuttgart: Kohlhammer.

Boreham, N., \& Morgan, C. (2004). A sociocultural analysis of organisational learning. Oxford Review of Education, 30(3), 307-325. doi:10.1080/0305498042000260467.

Cyert, R. M., \& March, J. G. (1992). A behavioral theory of the firm. Englewood Cliffs NJ: Prentice Hall.

Fahrenwald, C. (2009). Organisation und Erfahrung - Die Perspektive des modernen Wissensmanagements. In M. Göhlich, S. M. Weber \& S. Wolf (Hrsg.), Organisation und Erfahrung (S. 55-64). Wiesbaden: Springer VS.

Faulstich, P., Forneck, H.J., Grell, P., Häßner, K., Knoll, J., \& Springer, A. (2005). Lernwiderstand Lernumgebung - Lernberatung. Bielefeld: W. Bertelsmann.

Faulstich, P. (2013). Menschliches Lernen: Eine kritisch-pragmatistische Lerntheorie. Bielefeld: Transcript.

Fenwick, T., Edwards, R., \& Sawchuk, P. (2011). Emerging approaches to educational research. Tracing the sociomaterial. London, New York: Routledge.

Geißler, H. (2000). Organisationspädagogik: Umrisse einer neuen Herausforderung. München: Vahlen.

\footnotetext{
${ }^{4}$ In dem untersuchten Beispiel deutet viel darauf hin, dass insbesondere der Prozess der Abstimmung von Antriebs- und Inhaltsdimension ein tiefer gehender sein wird, der sich nicht allein auf den Ebenen abspielen wird, die bei Illeris mit materialem Lernen und (im Anschluss an Piaget) Assimilation beschrieben werden.
} 
Göhlich, M. (2007). Organisationales Lernen. In M. Göhlich, C. Wulf \& J. Zirfas (Hrsg.), Pädagogische Theorien des Lernens (S. 222-232). Weinheim, Basel: Beltz.

Göhlich, M., \& Zirfas, J. (2007). Lernen. Ein pädagogischer Grundbegriff. Stuttgart: Kohlhammer.

Göhlich, M., Weber, S. M., Schröer, A., et al. (2014). Forschungsmemorandum der Kommission Organisationspädagogik. Erziehungswissenschaft, 49, 94-105.

Gruschka, A. (2006). Pädagogische Theorien und empirische Forschung. In G. Wiesner, C. Zeuner \& H. J. Forneck (Hrsg.), Empirische Forschung und Theoriebildung in der Erwachsenenbildung (S. 34-48). Baltmannsweiler: Schneider Hohengehren.

Illeris, K. (2003). Towards a contemporary and comprehensive theory of learning. International Journal of Lifelong Education, 22(4), 396-406.

Illeris, K. (2004). Adult education and adult learning. Malabar, Florida: Krieger.

Illeris, K. (2006). Das „Lerndreieck“. Rahmenkonzept für ein übergreifendes Verständnis vom menschlichen Lernen. In E. Nuissl (Hrsg.), Vom Lernen zum Lehren. Lern- und Lehrforschung für die Weiterbildung (S. 29-41). Bielefeld: W. Bertelsmann.

Illeris, K. (2007). How we learn: Learning and non-learning in school and beyond. London: Routledge.

Illeris, K. (2010). Lernen verstehen. Bedingungen erfolgreichen Lernens. Bad Heilbrunn: Klinkhardt.

Kieser, A., \& Walgenbach, P. (2007). Organisation (5. Aufl.). Stuttgart: Schäffer-Poeschel.

Kolb, D. A. (1984). Experiential learning: Experience as the source of learning and development. Englewood Cliffs NJ: Prentice Hall.

Kromrey, H. (1998). Empirische Sozialforschung (8. Aufl.). Stuttgart: Leske und Budrich.

Leichthammer, K. (2000). Senge, P.M.: The fifth discipline. In K. Türk (Hrsg.), Hauptwerke der Organisationstheorie (S. 283-285). Wiesbaden: Westdeutscher Verlag.

Mandl, H., Kopp, B., \& Dworak, S. (2004). Aktuelle theoretische Ansätze und empirische Befunde im Bereich der Lehr-Lern-Forschung - Schwerpunkt Erwachsenenbildung. http://www.die-bonn.de/esprid/ dokumente/doc-2004/mand104_01.pdf. Zugegriffen: 10. Jan. 2017.

March, J. G., \& Olsen, J.P. (1976). Ambiguity and choice in organizations. Bergen: Universitetsforlaget.

March, J. G., \& Simon, H. A. (1958). Organizations. Camebridge, MA: Blackwell.

Mayring, P. (2010). Qualitative Inhaltsanalyse Grundlagen und Techniken (11. Aufl.). Weinheim: Beltz.

Meyer-Drawe, K. (2008). Diskurse des Lernens. München: Wilhelm Fink.

Mott, V.W. (2009). How we learn: Learning and non-learning in school and beyond. Adult Education Quarterly, 59(2), 177-179. Book Review: Illeris, K. (2007).

Pätzold, H. (2011). Learning and teaching in adult education. Contemporary theories. Opladen, Farmington Hills: Barbara Budrich.

Pätzold, H. (2015). Organisationstheorien in der Erwachsenenbildung: Rezeption und Nutzung. In M. Schemmann (Hrsg.), Organisationsforschung in der Erwachsenenbildung - Theorie, Methoden, Befunde (Bd. 38, S. 19-36). Wien, Köln, Weimar: Böhlau.

Pätzold, H. (2016). Akteur-Netzwerk-Theorie als Theorie und/oder Methode in der Organisationspädagogik. In A. Schröer, M. Göhlich, S.M. Weber \& H. Pätzold (Hrsg.), Organisation und Theorie (S. 295-304). Wiesbaden: Springer VS.

Pätzold, H. (2017). Bausteine einer Theorie der lernenden Non-Profit-Organisation. In A. Schröer, M. Göhlich \& S. M. Weber (Hrsg.), Organisation und dritter Sektor. Wiesbaden: Springer VS. i.Dr.

Schein, E. H. (2004). Organizational culture and leadership (3. Aufl.). San Francisco: Jossey-Bass.

Schiersmann, C., \& Thiel, H.-U. (2014). Organisationsentwicklung. Prinzipien und Strategien von Veränderungsprozessen (4. Aufl.). Wiesbaden: Springer VS.

Schrader, J., \& Berzbach, F. (2005). Empirische Lernforschung in der Erwachsenenbildung/Weiterbildung. http://www.die-bonn.de/esprid/dokumente/doc-2005/schrader05_01.pdf. Zugegriffen: 10. Jan. 2017.

Schreyögg, G., \& Geiger, D. (2016). Organisation: Grundlagen moderner Organisationsgestaltung: mit Fallstudien (60. Aufl.). Wiesbaden: Springer Gabler.

Schröer, A. (2011). Leadership in Nonprofit Organisationen. Programmatische Überlegungen zur Organisationspädagogischen Leadership-Forschung. In M. Göhlich, S. M. Weber, C. Schiersmann \& A. Schröer (Hrsg.), Organisation und Führung (S. 97-111). Wiesbaden: Springer VS.

Schurz, G. (2011). Evolution in Natur und Kultur: Eine Einführung in die verallgemeinerte Evolutionstheorie. Heidelberg: Spektrum.

Senge, P.M. (1990). The fifth discipline - the arts and practice of the learning organization. New York: Doubleday Currency.

Thomas, S. (2010). Ethnografie. In G. Mey \& K. Mruck (Hrsg.), Handbuch qualitative Forschung in der Psychologie (S. 524-537). Wiesbaden: Springer VS. 